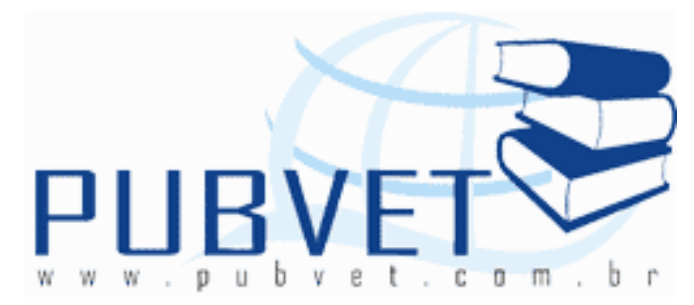

PUBVET, Publicações em Medicina Veterinária e Zootecnia.

\title{
Influência do período do ano e horário do dia sobre os parâmetros fisiológicos de ovelhas Santa Inês na microrregião do Alto Médio Gurguéia
}

Aline de Sousa Silva ${ }^{1}$, Tairon Pannunzio Dias e Silva ${ }^{2 *}$, Jaqueline da Silva Rocha ${ }^{1}$, Moisés Almeida Brito ${ }^{1}$, Naira Moura Alves ${ }^{3}$, Alécio Matos Pereira ${ }^{4}$

${ }^{1}$ Biólogos

${ }^{2}$ Médico Veterinário, Mestrando do Programa de Pós-Graduação em ZootecniaUFPI/CPCE

${ }^{3}$ Médica veterinária

${ }^{4}$ Médico Veterinário, Professor do Departamento de Medicina VeterináriaUFPI/CPCE

*tairon.mvet@gmail.com

\section{Resumo}

Vários fatores influenciam a produção animal, dentre eles os sistemas de produção e fatores climáticos podem provocar alterações fisiológicas e interferir na produtividade. Sendo assim, os autores objetivaram avaliar a influência do período do ano e horário do dia sobre os parâmetros fisiológicos de ovelhas Santa Inês na microrregião do Alto Médio Gurguéia. Foram utilizadas oito matrizes da raça Santa Inês distribuídas em um delineamento inteiramente casualizado em esquema fatorial $4 \times 2$, quatro períodos do dia, 78, 10-11, 14-15 e 17-18 h, e dois períodos: $\mathrm{PC}_{1}$ (quente e seco), outubro a 
novembro e $\mathrm{PC}_{2}$ (ameno e úmido), março a abril. Os parâmetros fisiológicos, frequência respiratória (FR), frequência cardíaca ( $F C$ ) e temperatura retal (TR), foram aferidos semanalmente, realizando-se quatro coletas por período, perfazendo um total de 08 coletas. Os parâmetros fisiológicos, FR, FC e TR diferiram $(P<0,05)$ entre período do ano e horário do dia, com utilização em maior intensidade, para todas as variáveis, no $\mathrm{PC}_{1}$ durante a tarde, principalmente às $17-18 \mathrm{~h}$, provavelmente devido ao aumento da temperatura ambiente. Houve influência de período do ano e horário do dia sobre os parâmetros fisiológicos das ovelhas da raça Santa Inês, com valores mais elevados no turno da tarde do período quente e seco.

Palavras-chave: bioclimatologia animal, efeito, estresse, fatores ambientais

\title{
Influence of time of year and time of day on the physiological parameters of Santa Inês ewes in the micro Upper Middle Gurguéia
}

\begin{abstract}
Several factors influence animal production, including production systems and climatic factors can cause physiological changes and interfere with productivity. Therefore, the authors aimed to evaluate the influence of the time of year and time of day on the physiological parameters of Santa Inês sheep in the microregion of the High Middle Gurguéia. We used eight arrays Santa Ines distributed in a completely randomized $4 \times 2$ factorial four periods of the day, $7-$ 8, 10-11, 14-15 and 17-18 h, and two periods: PC1 (hot and dry ), OctoberNovember and PC2 (mild and humid), March-April. Physiological parameters, respiratory rate $(R R)$, heart rate $(H R)$ and rectal temperature (RT) were measured weekly, performing four collections per period, a total of 08 collections. Physiological parameters, FR, HR and TR differ $(P<0.05)$ between season and time of day, using a higher intensity, for all variables in PC1 during the afternoon, especially at 17-18 h, probably due to increased temperature. Was no influence of time of year and time of day on the physiological
\end{abstract}


parameters of Santa Inês sheep, with higher values in the afternoon period of hot and dry.

Keywords: animal bioclimatology, effect, environmental factors, stress

\section{Introdução}

O sucesso dos sistemas de produção animal é mundialmente dependente do grau de eficiência econômico produtiva que se atinge, sendo importante salientar que essa eficiência não significa, propriamente, máxima produtividade. A cadeia produtiva de ovinos não é diferente e, portanto, precisa estar alinhada a essa tendência mundial (Menezes et al., 2010).

A atividade pecuária, sobretudo a criação de pequenos ruminantes, tem se constituído, ao longo tempo, a atividade básica das populações rurais distribuídas na região semi-árida do nordeste brasileiro muito em função das condições edafo-climáticas desfavoráveis, (Nóbrega et al., 2011).

Ainda não foi conseguido alternativa "milagrosa" para o semi-árido, suas potencialidades e formas de uso podem ser diferentes em função das particularidades específicas de cada uma delas. Condições ambientais adversas, como temperaturas elevadas verificadas durante grande parte do ano, implicando em exposição dos animais ao estresse crônico, possibilitando desequilíbrio endócrino e, consequentemente, alteração dos desempenhos produtivo e reprodutivo dos animais (Encarnação, 1989).

Dessa forma, o conhecimento das variações diárias e sazonais dos parâmetros fisiológicos permite a adoção de ajustes que promovam maior conforto aos animais e permitam uma produção pecuária de forma sustentável (Nóbrega et al., 2011).

$\mathrm{Na}$ busca do conforto do animal, o manejo ambiental surge como uma alternativa, em função da influência dos atributos climáticos em favorecer ou prejudicar o seu desempenho. Este manejo engloba as estratégias usadas para reduzir os problemas existentes na relação animal-ambiente. A eficiência produtiva é maior quando os animais estão em condições de 
termoneutralidade e não precisam acionar os mecanismos termorreguladores (Souza et al., 2005).

De posse das informações citadas acima, é importante destacar que o estresse calórico é um dos principais limitantes à produção animal nos trópicos, pois as elevadas temperaturas podem interferir no consumo de alimentos, no ganho de peso e nas taxas reprodutivas. Portanto, entre a alternativa de adequar as condições ambientais aos animais e a de selecionar animais capazes de produzir satisfatoriamente em ambientes adversos, essa última parece ser a solução mais prática (Cezar et al., 2004).

O desenvolvimento, no Brasil, de raças deslanadas como a Santa Inês apresenta-se como alternativa interessante para melhora da eficiência dos sistemas de produção de carne ovina (Costa Junior et al., 2006), animais que se destacam por serem altamente prolíficos, apresentam boa habilidade materna e adaptabilidade ao semiárido nordestino (Silva Sobrinho, 2006).

Diante do exposto os autores objetivaram avaliar a influência do período do ano e horário do dia sobre os parâmetros fisiológicos de ovelhas Santa Inês na microrregião do Alto Médio Gurguéia

\section{Material e métodos}

O experimento foi conduzido na Unidade de Pesquisa em Pequenos Ruminantes do campus Profa. Cinobelina Elvas da Universidade Federal do Piauí, Bom Jesus, Piauí, Brasil (09004'28" latitude sul e 44021'31" longitude oeste), a uma altitude de 277 metros.

Foram utilizadas oito matrizes, pluríparas, com peso médio de 43,76 \pm $5,03 \mathrm{Kg}$, da raça Santa Inês, mantidos em regime semi-extensivo, onde permaneciam das 07:00 às $16: 30 \mathrm{~h}$ em piquetes de capim andropogon (andropogon gayanus), sendo então, recolhidos em baias coletivas.

Os parâmetros fisiológicos como frequência respiratória (FR), frequência cardíaca (FC) e temperatura retal (TR), foram aferidos, nesta ordem, com os animais à sombra, nos seguintes horários: 7-8, 10-11, 14-15 e 17-18 h, uma vez a cada sete dias. Foram realizadas quatro coletas em cada período do ano: 
$\mathrm{PC}_{1}$ (quente e seco), outubro a novembro e $\mathrm{PC}_{2}$ (ameno e úmido), março a abril, perfazendo um total de 08 coletas.

A frequência respiratória foi avaliada por contagem dos movimentos respiratórios por minuto ( $\mathrm{mov} / \mathrm{min})$, mediante a observação direta dos movimentos do flanco esquerdo. A frequência cardíaca, em batimentos por minuto (bat/min), foi obtida com a utilização de um estetoscópio posicionado entre o terceiro e quarto espaço intercostal, à altura da articulação costocondral, durante um minuto, na sequência foi avaliado a temperatura retal por meio de termômetro digital até o disparo do sonorizador de acordo com as metodologias utilizadas por Sousa Júnior et al. (2008).

A temperatura ambiente (TA) e umidade relativa (UR) foram medidas com auxílio de termo-higrômetro (Incoterm, Porto Alegre, Brasil) e a temperatura de globo negro (TGN), mediante um globo-termômetro (termômetro Inconterm 0 a $100^{\circ} \mathrm{C}$ inserido a um globo negro de $150 \mathrm{~mm}$ de diâmetro), instalados à altura de $55 \mathrm{~cm}$ do solo, que corresponde à altura média aproximada dos animais. Para o ITU foi utilizada a equação proposta por Thom (1959): ITU: $(0,8 \times \mathrm{T}+(\mathrm{UR}(\%) / 100) \times(\mathrm{T}-14,4)+46,4)$, onde: $\mathrm{T}=$ temperatura do $\operatorname{ar}^{\circ} \mathrm{C}$ e UR $=$ umidade relativa do ar.

O delineamento estatístico utilizado foi o inteiramente casualizado em esquema fatorial $4 \times 2$ (quatro horários e duas épocas), com oito repetições, utilizando-se o logiciário estatístico SAS (SAS, 1999), a comparação entre as médias pelo teste de Tukey a $5 \%$ de probabilidade.

\section{Resultados e discussão}

A Tabela 1 apresenta os valores médios das variáveis ambientais e índice de temperatura de globo e umidade registrados nos diferentes períodos climáticos ( $\mathrm{PC}_{1}$, quente e seco e $\mathrm{PC}_{2}$, ameno e úmido). De acordo com os valores médios da $T A$, constatou-se interação $(P<0,05)$ entre os períodos do ano em que, no período quente e seco a TA apresentou valor superior ao período ameno e úmido. Segundo Lu (1989), a temperatura ambiente de $30^{\circ} \mathrm{C}$ 
SILVA, A.S. et al. Influência do período do ano e horário do dia sobre os parâmetros fisiológicos de ovelhas Santa Inês na microrregião do Alto Médio Gurguéia. PUBVET, Londrina, V. 7, N. 21, Ed. 244, Art. 1612, Novembro, 2013.

é como o limite superior da zona de termoneutralidade para caprinos, que poderia ser usada como referência também para ovinos deslanados.

Temperatura ambiental elevada aliada a alta incidência de radiação solar geram consequências no sistema de produção, principalmente na região semiárida do nordeste, com animais criados a pasto, podendo acarretar estresse calórico aos animais, ocasionando declínio na produção (Silva et a., 2012).

Tabela 1. Médias das variáveis ambientais e índice de temperatura e umidade (ITU) registrados nos diferentes períodos climáticos e horários do dia durante o período experimental.

\begin{tabular}{|c|c|c|c|c|}
\hline \multirow{2}{*}{$\begin{array}{c}\text { Variáveis } \\
\text { ambientais }\end{array}$} & \multicolumn{4}{|c|}{ Períodos climáticos } \\
\hline & \multicolumn{2}{|c|}{$\begin{array}{l}\text { Quente e seco } \\
\left(\mathrm{PC}_{1}\right)\end{array}$} & \multicolumn{2}{|c|}{$\begin{array}{c}\text { Ameno e úmido } \\
\left(\mathrm{PC}_{2}\right)\end{array}$} \\
\hline $\begin{array}{l}\text { TA }\left({ }^{\circ} \mathrm{C}\right) \\
\text { UR }(\%)\end{array}$ & \multicolumn{2}{|c|}{$\begin{array}{l}31,28 \pm 11,67^{\mathrm{A}} \\
5463 \pm 2147^{\mathrm{A}}\end{array}$} & \multicolumn{2}{|c|}{$26,63 \pm 8,63^{\mathrm{B}}$} \\
\hline $\begin{array}{l}\text { TGN }\left({ }^{\circ} \mathrm{C}\right) \\
\text { ITU }\end{array}$ & \multicolumn{2}{|c|}{$\begin{array}{l}32.81 \pm 4.66^{\mathrm{A}} \\
78,99 \pm 5,90^{\mathrm{A}}\end{array}$} & \multicolumn{2}{|c|}{$\begin{array}{l}28.44 \pm 2.01^{B} \\
74.62 \pm 2.69^{B}\end{array}$} \\
\hline \multirow{2}{*}{ ITU } & \multicolumn{4}{|c|}{ Horários do dia } \\
\hline & $7-8 \mathrm{~h}$ & $10-11 \mathrm{~h}$ & $14-15 \mathrm{~h}$ & $17-18 \mathrm{~h}$ \\
\hline TA $\left({ }^{\circ} \mathrm{C}\right)$ & $26,06 \pm 14,82^{\mathrm{A}}$ & $29,44 \pm 8,88^{\mathrm{B}}$ & $30,56 \pm 13,09^{B}$ & $29,75 \pm 10,56^{\mathrm{B}}$ \\
\hline UR (\%) & $74,87 \pm 14,82^{\mathrm{A}}$ & $66,50 \pm 20,02^{B}$ & $64,25 \pm 25,39^{B}$ & $67,00 \pm 27,19^{B}$ \\
\hline TGN ( $\left.{ }^{\circ} \mathrm{C}\right)$ & $28,63 \pm 5,62^{A}$ & $31,75 \pm 9,09^{\mathrm{B}}$ & $32,06 \pm 12,65^{B}$ & $30,06 \pm 9,04^{\mathrm{B}}$ \\
\hline ITGU & $74,81 \pm 7,51^{\mathrm{A}}$ & $77,93 \pm 3,71^{\mathrm{B}}$ & $78,24 \pm 5,18^{\mathrm{B}}$ & $76,24 \pm 3,55^{\mathrm{B}}$ \\
\hline
\end{tabular}

Com base nos dados de umidade relativa do ar, pode-se verificar superioridade no periodo ameno e úmido contrastando com a TA no período quente e seco, e corroborando com dados de (Silva et al., 2012), que relata uma relação direta e inversamente proporcional entre essas variáveis climatológicas.

A umidade relativa (UR) nos diferentes horários esteve dentro da faixa de conforto térmico para as ovelhas, que segundo Baeta \& Sousa (2010), deve estar entre 50 e $80 \%$.

Analisando-se o ITU, nota-se que, no período quente e seco os valores encontram-se acima da situação de conforto segundo Armstrong (1994) e, sob 
SILVA, A.S. et al. Influência do período do ano e horário do dia sobre os parâmetros fisiológicos de ovelhas Santa Inês na microrregião do Alto Médio Gurguéia. PUBVET, Londrina, V. 7, N. 21, Ed. 244, Art. 1612, Novembro, 2013.

condição mais crítica no turno da tarde no horário de 14-15 h, caracterizando situação de estresse moderado.

O estresse térmico foi classificado por Armstrong (1994) de acordo com o ITU em ameno ou brando (72 a 78), moderado (79 a 88) e, severo (89 a 98). Ainda ressaltou que valor abaixo de 72 , não caracteriza estresse térmico.

Na tabela 2 estão descritas as médias dos parâmetros fisiológicos das ovelhas durante o período experimental.

Tabela 2. Médias dos parâmetros fisiológicos de ovelhas da raça Santa Inês nos diferentes períodos $\left(\mathrm{PC}_{1}\right.$ e $\left.\mathrm{PC}_{2}\right)$ e diferentes horários do dia.

\begin{tabular}{|c|c|c|c|c|c|c|}
\hline \multirow{4}{*}{$\begin{array}{l}\text { Horários } \\
7-8 \mathrm{~h}\end{array}$} & \multicolumn{6}{|c|}{ Variáveis fisiológicas } \\
\hline & \multicolumn{3}{|c|}{$\begin{array}{c}\text { Quente e seco } \\
\left(\mathrm{PC}_{1}\right)\end{array}$} & \multicolumn{3}{|c|}{$\begin{array}{c}\text { Ameno e úmido } \\
\left(\mathrm{PC}_{2}\right)\end{array}$} \\
\hline & FR & FC & TR & FR & FC & $\overline{T R}$ \\
\hline & $38,07^{d, A}$ & $91,50^{c, A}$ & $38,75^{b, A}$ & $30,46^{d, B}$ & $74,32^{c, B}$ & $37,98^{\mathrm{c}, \mathrm{B}}$ \\
\hline $10-11 \mathrm{~h}$ & $50,36^{b, A}$ & $102,93^{a, A}$ & $38,99^{\mathrm{b}, \mathrm{A}}$ & $38,14^{\mathrm{c}, \mathrm{B}}$ & $88,00^{a, B}$ & $38,34^{\mathrm{b}, \mathrm{B}}$ \\
\hline $14-15 h$ & $53,78^{a, A}$ & $97,28^{b, A}$ & $38,78^{b, A}$ & $40,07^{\mathrm{b}, \mathrm{B}}$ & $76,18^{\mathrm{b}, \mathrm{B}}$ & $38,41^{\mathrm{b}, \mathrm{B}}$ \\
\hline $17-18 \mathrm{~h}$ & $45,37^{\mathrm{C}, \mathrm{A}}$ & $102,57^{a, A}$ & $39,29^{a, A}$ & $44,72^{a, B}$ & $72,96^{\mathrm{d}, \mathrm{B}}$ & $38,96^{a, B}$ \\
\hline
\end{tabular}

*letras diferentes minúsculas nas linhas e maiúsculas nas colunas diferem entre si pelo teste Tukey a 5\% de significância.

Houve interação significativa $(P<0,05)$ entre os fatores época do ano e horários do dia para todos os parâmetros (FR, FR e TR)

Percebe-se que os parâmetros fisiológicos estudados apresentaram médias superiores no turno da tarde, principalmente entre 14 e $15 \mathrm{~h}$, o que pode ser explicado pelos valores mais elevados dos parâmetros térmicos ambientais estudados durante o período experimental (Tabela 1). Estes resultados se assemelham aos de Cesar et al. (2004) e Neiva et al. (2004).

O turno da tarde caracteriza-se pela maior incidência de radiação solar, onde as ovelhas necessitaram utilizar em maior intensidade os mecanismos de dissipação de calor, buscando manter a homeotermia (Baêta \& Sousa, 2010).

A FR foi significativamente maior $(P<0,05)$ no horário de 14 e $15 \mathrm{~h}$. Esse comportamento corrobora com Sousa Júnior et al. (2008) citando que, quando acorre uma elevação acentuada na temperatura ambiente, os mecanismos termorregulatórios são acionados, aumentando a perda de calor na forma 
SILVA, A.S. et al. Influência do período do ano e horário do dia sobre os parâmetros fisiológicos de ovelhas Santa Inês na microrregião do Alto Médio Gurguéia. PUBVET, Londrina, V. 7, N. 21, Ed. 244, Art. 1612, Novembro, 2013.

insensível, através da sudorese, e aumento da FR na tentativa de dissipação de calor. Segundo Silva et al. (2012) a elevação da temperatura ambiente com consequente acúmulo de calor pelos animais e a incapacidade de dissipação do calor retido promovem um decréscimo no desempenho produtivo dos animais.

Swenson \& Reece (1996) reportam que a frequência respiratória (FR) média dos ovinos é em torno de 16 a $34 \mathrm{mov} / \mathrm{min}$ (movimentos/minuto), valores esses inferiores às médias obtidas neste trabalho. A FR em ruminantes, em ambientes termoneutros oscila entre 24 a $36 \mathrm{mov} / \mathrm{min}$ e acima da temperatura crítica superior esses valores podem estar várias vezes aumentados (Silva Sobrinho, 2006).

Quando se avalia a FC entre os turnos e os períodos climáticos, houve diferença $(P<0,05)$ com superioridade encontrada para a $P C_{1}$, com valores mais elevados entre 10 e $11 \mathrm{~h}$, provavelmente devido à alta incidência de radiação solar. Contudo, os valores em ambos os turnos e períodos climáticos encontram-se dentro dos padrões de referencia descrito para a espécie (60$110 \mathrm{bat} / \mathrm{min}$ ) de acordo com Souza et al. (2011).

Quando se analisa a TR entre os fatores em estudo, verificaram-se valores superiores para o período quente e seco, principalmente no horário entre $17-16 \mathrm{~h}\left(39,29^{\circ} \mathrm{C}\right)$, em consequência do acúmulo de calor ao longo do dia. Porém, segundo Silva (2000), a temperatura retal de ovinos pode estar na faixa de $38,9^{\circ} \mathrm{C}$ a $40,5^{\circ} \mathrm{C}$, o que representa estar dentro da zona de conforto térmico.

A temperatura retal é o resultado entre a energia térmica produzida e a energia térmica dissipada (Legates et al., 1991). Um aumento da temperatura retal significa que o animal está estocando calor, e se este não esta dissipando o estresse calórico manifesta-se (Swenson \& Reece, 1996).

\section{Conclusões}

Houve influência de período do ano e horário do dia sobre os parâmetros fisiológicos das ovelhas da raça Santa Inês, com valores mais elevados no período quente e seco e no turno da tarde. 


\section{Referências bibliográficas}

Armstrong, D.V. Heat stress interaction with shade and cooling. Journal of Dairy Science, v.77, p.2044-2050. 1994.

BAÊTA, F.C.; SOUZA, C.F. Ambiência em edificações rurais: Conforto animal. $2^{a}$ ed. Viçosa: UFV. 2010. 246p.

CESAR, M.F.; SOUZA, B.B.; SOUZA, W.H. Avaliação de parâmetros fisiológicos de ovinos Dorper, Santa inês e seus mestiços perante condições climáticas do trópico semi-árido nordestino. Ciência e Agrotecnologia, v.28, n.3, p.614-620, 2004.

ENCARNAÇÃO, R.O. Estresse e produção animal. In: Ciclo Internacional de Palestras Sobre Bioclimatologia Animal, 1989, Jaboticabal. Anais... Jaboticabal: FUNEP, 1989. p.111-129, 1989.

LEGATES, J.E.; FARTHING, B.R.; CASADY, R.B. Body temperature and respiratory rate of lactating dairy cattle under field and chamber conditions. Journal Dairy Science, v.74, p.2491-2500, 1991.

Lu, C.D. Effects of heat stress on goat production. Small Ruminant Research, v.2, n.2, p.151-162, 1989.

MENEZES, G.R.O.; TORRES, R.A.; SARMENTO, J.L.R.; RODRIGUES, M.T.; MELO, A.L.P.; SILVA, F.G.; BRITO, L.F. Avaliação de medidas da persistência da lactação de cabras da raça Saanen sob modelo de regressão aleatória. Revista Brasileira de Zootecnia, v.39, n.8, p.1691$1698,2010$.

NEIVA, J.N.M.; TEIXEIRA, M.; TURCO, S.H.N. Efeito do estresse climático sobre os parâmetros produtivos e fisiológicos de ovinos Santa Inês mantidos em confinamento na região litorânea do nordeste do Brasil. Revista Brasileira de Zootecnia, v.33, n.3, p.668-678, 2004.

NEIVA, J.N.M.; TEIXEIRA, M.; TURCO, S.H.N.; OLIVEIRA, S.M.P.; MOURA, A.A.A.N. Efeito do estresse climático sobre os parâmetros produtivos e fisiológicos de ovinos Santa Inês mantidos em confinamento na região litorânea do nordeste do Brasil. Revista Brasileira de Zootecnia, v.33, n.3, p.668-678, 2004.

NÓBREGA, G.H.; SILVA, E.M.N.; SOUZA, B.B.; MANGUEIRA, J.M.A produção animal sob a influência do ambiente nas condições do semiárido nordestino. Revista verde de agroecologia e desenvolvimento sustentável. V.06, n.01, p.67-73, 2011.

NRC, Nutrient Requirements of Small Ruminants: Sheep, Goats, Cervids and New World Camelids. National Academy Press, Washington, DC, 2007. 384p.

ROBERTO, J.V.B.; SOUZA, B.B. Fatores ambientais, nutricionais e de manejo e índices de conforto térmico na produção de ruminantes no semiárido. Revista Verde de agroecologia e desenvolvimento sustentável, v.6, n.2, p.08-13, 2011.

SAS - Statistical Analysis Systems. 1999. User's guide: SAS Institute Inc. 1999.

SILVA SOBRINHO, A.G. Criação de ovinos. 3. ed. FUNEP: Jaboticabal. 2006. 302p. 
SILVA, G.A.; SOUZA, B.B.; ALFARO, C.E.P.; NETO, J.A.; AZEVEDO, S.A.; SILVA, E.M.N.; SILVA, R.M.N. Influência da dieta com diferentes níveis de lipídeo e proteína na resposta fisiológica e hematológica de reprodutores caprinos sob estresse térmico. Ciência e Agrotecnologia, v.30, n.1, p.154-161, 2006.

SILVA, T.P.D.; OLIVEIRA, R.G.; SOUSA JÚNIOR, S.C.; SANTOS, K.R. Efeito da exposição à radiação solar sobre parâmetros fisiológicos e estimativa do declínio na produção de leite de vacas mestiças (Holandês $X$ Gir) no sul do estado do Piauí. Comunicata Scientiae, v.3, n.4, p.299-305, 2012.

SOUSA JÚNIOR, S.C.; MORAIS, D.A.E.F.; VASCONCELOS, A.M.; NERY, K.M.; MORAIS, J.H.G.; GUILHERMINO, M.M. Características Termorreguladoras de Caprinos, Ovinos e Bovinos em Diferentes Épocas do Ano em Região Semi-Árida. Revista Científica de Produção Animal, v.10, n.2, p.127-137, 2008.

SOUZA, B.B.; ASSIS, D.Y.C.; SILVA NETO, F.L.; ROBERTO, J.V.B.; MARQUES, B.A.A. Efeito do clima e da dieta sobre os parâmetros fisiológicos e hematológicos de cabras da raça saanen em confinamento no sertão paraibano. Revista Verde, v.6, n.1, p.77-82, 2011.

SOUZA, E.D.; SOUZA, B.B.; SOUZA, W.H. Determinação dos parâmetros fisiológicos e gradiente térmico de diferentes grupos genéticos de caprinos no Semi-Árido. Ciência e Agrotecnologia, v.29, n.1, p.177-184. 2005.

SWENSON, M.J.; REECE, W.O. Dukes Fisiologia dos animais domésticos. 11 ed. Guanabara Koogan: Rio de Janeiro; 1996, 856p.

THOM, E.C. 1959. The discomfort index. Weather wise: 57-59p. 